\title{
Synthesis of Morpholine Containing Sulfonamides: Introduction of Morpholine Moiety on Amine Functional Group
}

\author{
Singh, D. and Bansal, G.* \\ Department of Pharmaceutical Sciences \& Drug Research, \\ Punjabi University, Patiala.
}

Received 17 March 2004; Accepted 1 May 2004

\begin{abstract}
Sulfonamides have been the center of drug structures as this group is quite stable $\&$ well tolerated in human beings. The synthesis of these structures was started in search of new pharmacological active reagents. These compounds are being tested for the desired activity (ICAM-1/LFA-1 Interaction inhibitors as anti-adhesion therapeutic agents), the biological activity \& structure activity relationship will be published elsewhere. Synthesis of morpholine moiety from amino group is done by using reagent 2-chloroethanol.
\end{abstract}

Key words: Sulfonamides, Morpholine, anti-adhesion molecules.

\section{Introduction}

Sulfonamides have been the center of drug structures as this group is quite stable $\&$ well tolerated in human beings. The synthesis of these structures was started in search of new pharmacological active reagents. These compounds are being tested for the desired activity (ICAM-1/LFA-1 Interaction inhibitors as anti-adhesion therapeutic agents ${ }^{1}$ ), the biological activity $\&$ structure activity relationship will be published elsewhere.

Synthesis of sulfonamide ${ }^{2}$ based target compounds involved coupling reactions under different conditions and reduction of the nitro compounds. Elucidation of the structures is done on the basis of ${ }^{1} \mathrm{H}$ NMR, FTIR \& confirmed with MS analysis.

Synthetic Schemes

The synthetic scheme employed involves a key intermediate sulfonamide $\mathrm{GD}_{2}$ are summarized as follows. 


\section{Scheme 1}

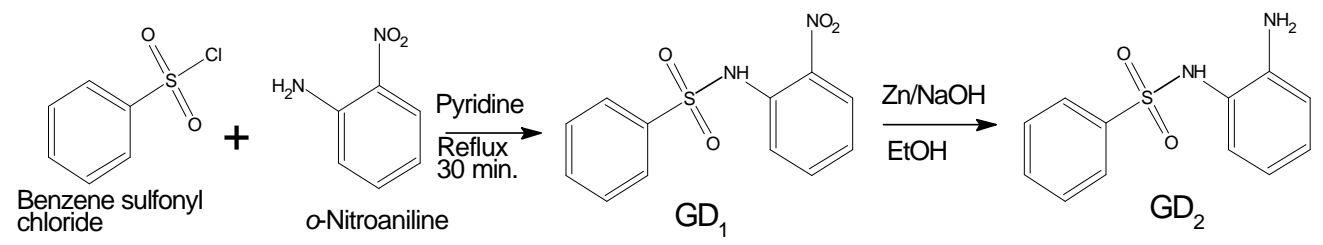

Scheme 1 depicts the synthesis of $\mathrm{GD}_{1}$ (Sulfonamide). Sulfonamide synthesis involve the reaction of sulphonyl chloride with amines either in acetone or in basic aqueous media. ${ }^{3}$ Feebly basic amines, e.g. the nitroanilines, generally react so slow with benzene sulfonyl chloride that most of the acid chloride is hydrolyzed by the aqueous alkali before a reasonable yield of the sulfonamide is produced; indeed, o-nitroaniline gives little or no sulfonamide under normal reaction conditions. ${ }^{3}$ When reaction was carried out in pyridine, the yields were high. The reaction was observed to be time dependent. More than 30 min. resulted a di-sulfonyl substituted nitroaniline with similar $R_{f}$ value in TLC, but being insoluble in rectified spirit. This formed the basis of purification by differential solubility.

Reduction of nitro group in $\mathrm{GD}_{1}$ is done by using $\mathrm{Z} / \mathrm{NaOH}$ reduction method. ${ }^{3}$ This method was preferred over tin/ $\mathrm{HCl}$ or stannous chloride in acid because of better yield and is less time consuming. TLC plate showed four spots, which gave three spots in diethyl ether solution (impurities). Hence, diethyl ether washing was selected to purify the product. Amount of zinc was increased more than normal to reduce the impurities and time of reaction. This reaction was used in all other reductions and worked well with high yields. The only precaution to be observed is that the ratio of $\mathrm{NaOH} \&$ rectified spirit should be kept constant irrespective of the starting compound taken.

\section{Scheme 2}

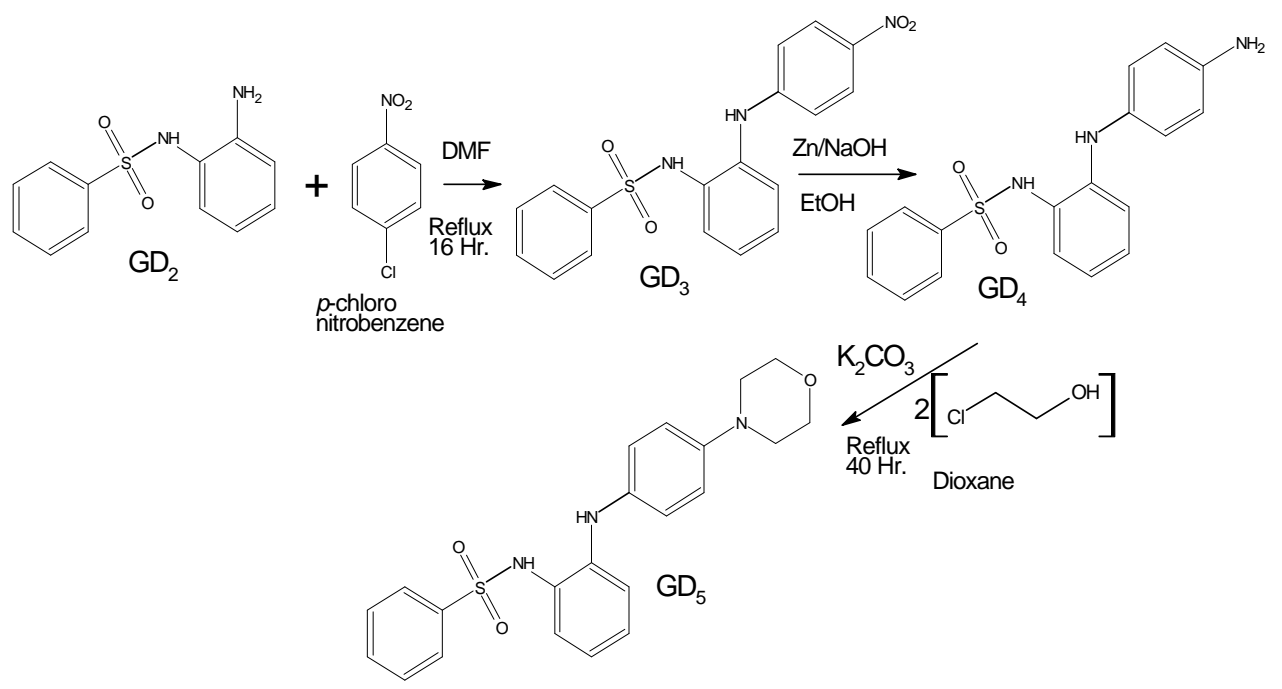


Scheme 2 indicate the formation of $\mathrm{GD}_{3}$, a simple coupling involving loss of $\mathrm{HC}$ and the reaction was completed with refluxing in N,N-dimethyl formamide for $16 \mathrm{Hr}$. DMF is chosen as a high boiling organic solvent, miscible with water. On pouring this solution in ice water $\mathrm{GD}_{3}$ precipitated out.

Following this $\mathrm{GD}_{3}$ is reduced to $\mathrm{GD}_{4}$ using standardized $\mathrm{Zn} / \mathrm{NaOH}$ reduction procedure as for $\mathrm{GD}_{2}$. Following this was coupling of $\mathrm{GD}_{4}$ with 2-chloroethanol ${ }^{4}$ to form $\mathrm{GD}_{5}$. Amine is replaced by morpholine functionality. Amine can be diazotised and then morpholine can replace the diazo group. The reaction with 2-chloroethanol was designed using the fact that alkyl chloride couple with aromatic amines at temperature $58^{\circ} \mathrm{C}$ with stirring in dioxane in $40 \mathrm{Hr}$. The time was kept same but conditions made more severe involving more potassium carbonate and refluxing conditions. The reactant spot $\left(\mathrm{GD}_{4}\right)$ on $\mathrm{TLC}$ disappeared from reaction mixture after $11 \mathrm{Hrs}$. As we were not sure of cyclization, the reaction was continued for $40 \mathrm{Hrs}$. The worked up reaction showed no intermediate product. Reduction of reaction time was not attempted.

Scheme 3

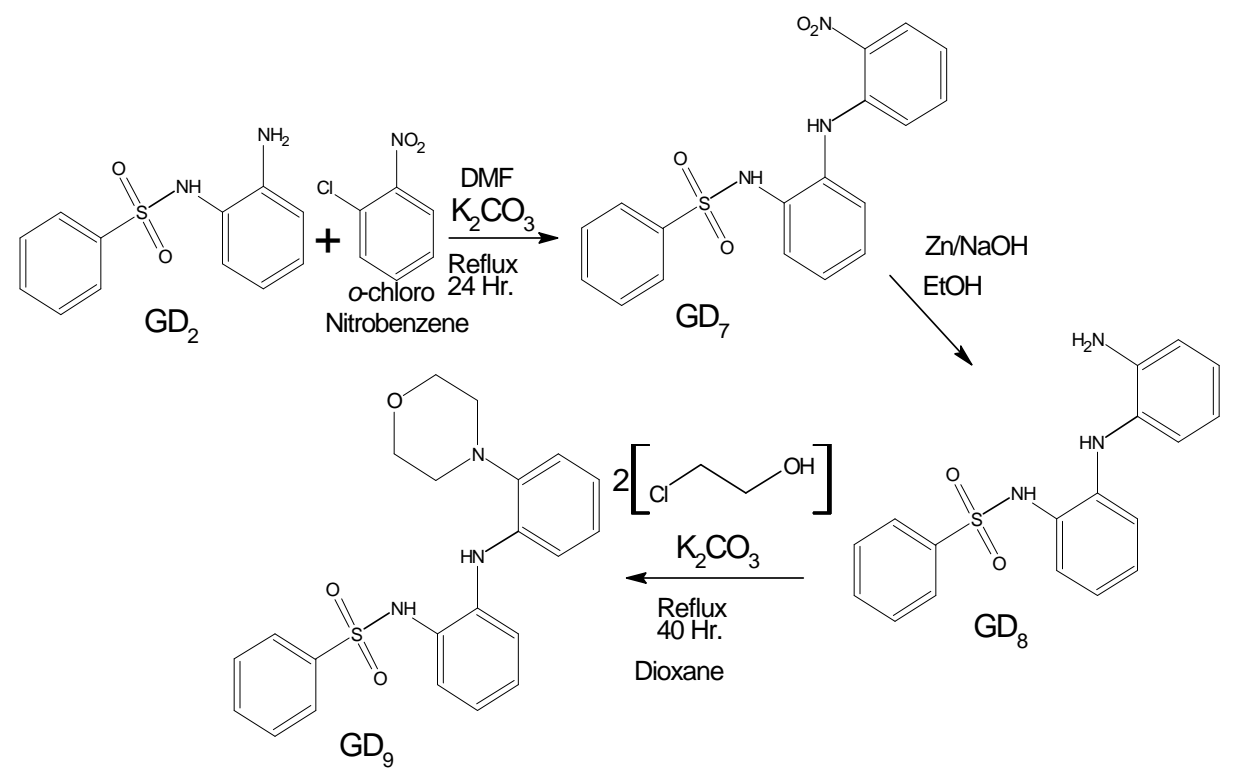

Scheme 3 depicts the synthesis of $\mathrm{GD}_{9}$. Ortho-Chloro nitrobenzene being more crow ded requires severe conditions to couple with $\mathrm{GD}_{2}$. Addition of potassium carbonate and increase in time to $24 \mathrm{Hrs}$ served the purpose. ${ }^{3}$ The reaction followed a peculiar pattern in that if severe refluxing was done, the product isolated was containing an oily material (yellow in color). Oil mixed with product and was not separable with solubility technique. It could only be separated by heating, as the liquid evaporated easily and leaving solid product behind, but care was taken not to char product and avoid constant heating to stop bumping of the whole product. Whole problem was avoided with controlled refluxing conditions. Other reactions were same as in scheme 2. 
Scheme 4

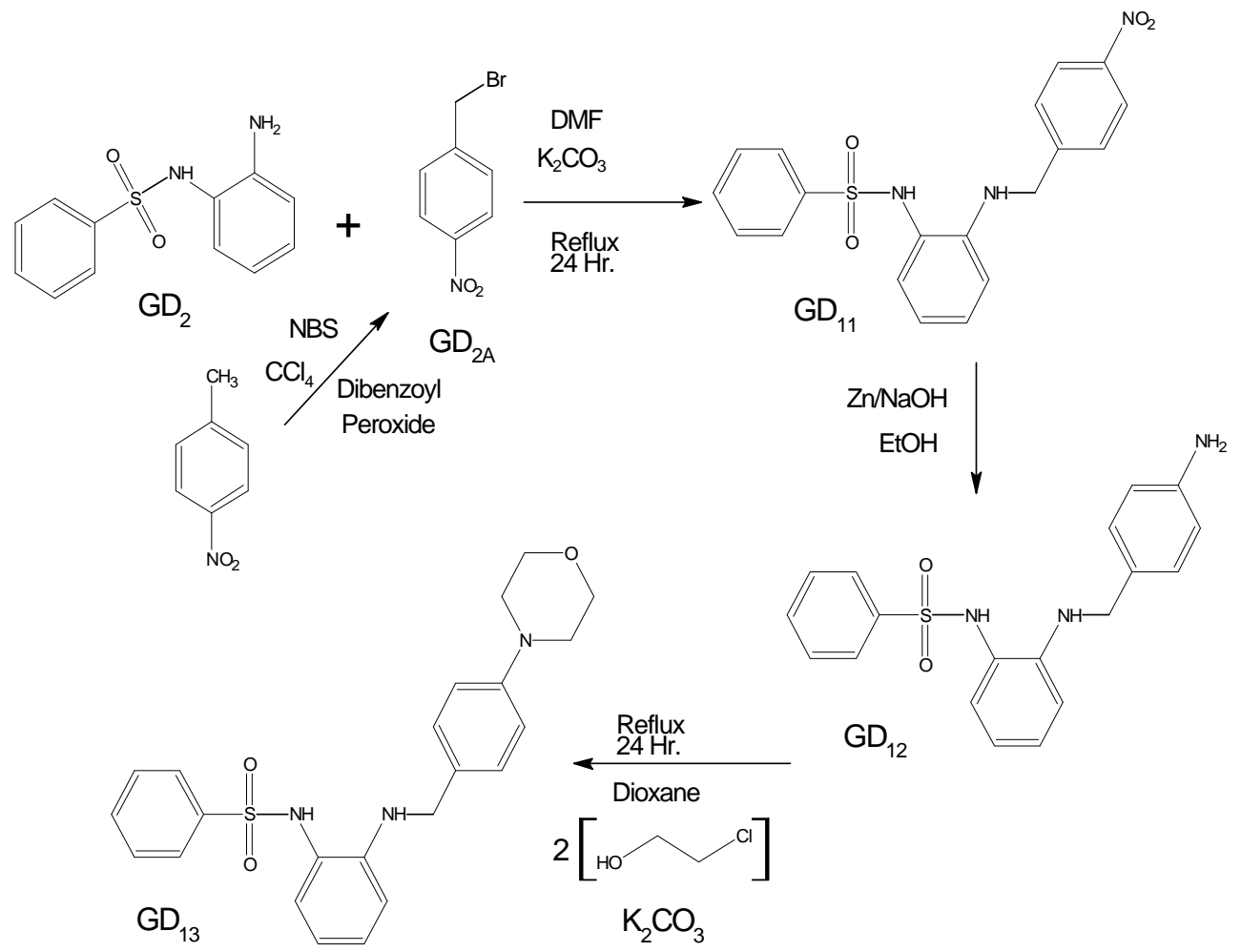

Scheme 4 indicates formation of $\mathrm{GD}_{13}$. The formation of $\mathrm{GD}_{2 \mathrm{~A}}$, from 4-nitro toluene was done by bromination, using standard N-Bromo succinimide. ${ }^{3}$ Dibenzoyl peroxide was used as free radical chain initiator in $\mathrm{Ca}_{4}$, forming a pure product. $\mathrm{GD}_{2 \mathrm{~A}}$ being alkyl halide also need severe conditions like in scheme 3. Other reactions were same as in scheme 3.

\section{Obervation Data}

Synthesised compounds were purified and their structure was elucidated based on $1 \mathrm{H}-\mathrm{NMR}(T a b l e ~ 3) \&$ IR data (Table 2). The structures were further confirmed by Mass Spectra (data not shown) 


\begin{tabular}{|c|c|c|c|c|}
\hline Name/Compd. No. & $\begin{array}{l}\text { Sol. in } \\
\text { Water }\end{array}$ & $\begin{array}{l}\text { Recrys. } \\
\text { Solvent }\end{array}$ & $\begin{array}{l}\text { Melting } \\
\text { Point }{ }^{\circ} \mathrm{C}\end{array}$ & $\%$ yield \\
\hline $\begin{array}{l}\text { N-(2-nitrophenyl) benzene } \\
\text { sulfonamide } \quad\left(G_{1}\right)\end{array}$ & - & $\mathrm{C}_{2} \mathrm{H}_{5} \mathrm{OH}$ & 107-108 & 89.94 \\
\hline $\begin{array}{l}\mathrm{N} \text {-(2-aminophenyl) benzene } \\
\text { sulphonamide }\left(\mathrm{GD}_{2}\right)\end{array}$ & + & $\mathrm{CH}_{3} \mathrm{OH}$ & $121-122$ & 96.52 \\
\hline $\begin{array}{l}\mathrm{N} \text { - }\{2-[(4-\text { nitrophenyl) amino }] \\
\text { phenyl\}benzenesulfonamide }\left(\mathrm{GD}_{3}\right)\end{array}$ & - & $\mathrm{CH}_{3} \mathrm{OH}$ & $162-163$ & 54.2 \\
\hline $\begin{array}{l}\mathrm{N}-\{2 \text { - }[(4 \text {-aminophenyl) amino }] \text { phenyl }\} \\
\text { benzenesulfonamide }\left(\mathrm{GD}_{4}\right)\end{array}$ & + & $\mathrm{CHO}_{3}$ & 184-185 & 89.2 \\
\hline $\begin{array}{l}\mathrm{N} \text { - }\{2 \text {-[4(-4-morpholino)anilino] phenyl\} } \\
\text { benzenesulfonamide }\left(\mathrm{GD}_{5}\right)\end{array}$ & - & $\mathrm{CH}_{3} \mathrm{OH}$ & $209-210$ & 88.71 \\
\hline 1-(bromomethyl)-4-nitrobenzene $\left(\mathrm{GD}_{2 \mathrm{~A}}\right)$ & - & $\mathrm{C}_{2} \mathrm{H}_{5} \mathrm{OH}$ & $100-101$ & 81.4 \\
\hline $\begin{array}{l}\mathrm{N}-\{2-[(2 \text {-nitrophenyl) amino }] \\
\text { phenyl\}benzenesulfonamide }\left(\mathrm{GD}_{7}\right)\end{array}$ & - & $\mathrm{CH}_{3} \mathrm{OH}$ & 148-149 & 53.09 \\
\hline $\begin{array}{l}\mathrm{N} \text { - }\{2-[(2 \text {-aminophenyl }) \text { amino }] \text { phenyl }\} \\
\text { benzenesulfonamide }\left(\mathrm{GD}_{8}\right)\end{array}$ & + & $\mathrm{CHO}_{3}$ & 168-169 & 88.7 \\
\hline $\begin{array}{l}\mathrm{N}-\{2-[2-(-4-\text { morpholino }) \text { anilino }] \\
\text { phenyl\} benzenesulfonamide }\left(\mathrm{GD}_{9}\right)\end{array}$ & - & $\mathrm{CH}_{3} \mathrm{OH}$ & 194-195 & 79.2 \\
\hline $\begin{array}{l}\mathrm{N}-\{2-[(4-\text { nitrobenzyl) amino }] \\
\text { phenyl\}benzenesulfonamide }\left(\mathrm{GD}_{11}\right)\end{array}$ & - & $\mathrm{CH}_{3} \mathrm{OH}$ & 179-180 & 50.12 \\
\hline $\begin{array}{l}\mathrm{N}-\{2-[(2 \text { aminobenzyl) amino }] \\
\text { phenyl }\} \text { benzenesulphonamide }\left(\mathrm{GD}_{12}\right)\end{array}$ & + & $\mathrm{CHO}_{3}$ & 197-198 & 90 \\
\hline $\begin{array}{l}\mathrm{N}-\{2-[4-(-4-\text { morpholino }) \text { benzylamino }] \\
\text { phenyl\} benzenesulfonamide }\left(\mathrm{GD}_{13}\right)\end{array}$ & - & $\mathrm{CH}_{3} \mathrm{OH}$ & $219-220$ & 83.45 \\
\hline
\end{tabular}

Table 2 IR Data (KBr Pellets)

\begin{tabular}{|c|c|c|c|c|c|c|c|c|c|c|c|c|}
\hline $\begin{array}{c}\text { Comp. } \mathrm{GD}_{\mathrm{N}} / \mathrm{N} \rightarrow \\
\text { IR Frequency present }\end{array}$ & 1 & 2 & 3 & 4 & 5 & $2 A$ & 7 & 8 & 9 & 11 & 12 & 13 \\
\hline $\begin{array}{c}\mathrm{N}-\mathrm{H} \text { str. } \\
\text { (sulphonamide) }\end{array}$ & + & + & + & + & + & - & + & + & + & + & + & + \\
\hline $\begin{array}{l}\text { Asym./Sym. N-H str. } \\
\text { (Amine) }\end{array}$ & - & + & + & + & + & - & + & + & + & + & + & + \\
\hline Ar C-H str. (asym/Sym) & + & + & + & + & + & + & + & + & + & + & + & + \\
\hline Asym./Sym. $\mathrm{N}(=\mathrm{O})_{2}$ str. & + & - & + & - & - & + & + & - & - & + & - & - \\
\hline $\begin{array}{l}\text { Asym./Sym.S }(=0)_{2} \\
\text { stretching }\end{array}$ & + & + & + & + & + & - & + & + & + & + & + & + \\
\hline Asym/SymC-O-Estr. & - & - & - & - & + & - & - & - & + & - & - & + \\
\hline
\end{tabular}


Table 3 NMR DATA $\left(\mathrm{CD}_{3}\right)$

\begin{tabular}{|c|}
\hline \\
\hline $\begin{array}{l}\mathrm{GD}_{1} \Rightarrow \delta 9.86: 1 \mathrm{H}, \mathrm{s} ; \delta 8.11: 1 \mathrm{H}, \mathrm{dd}(\mathrm{J}=8.2 ; 1.3) ; \delta 7.97: 1 \mathrm{H}, \mathrm{d}(\mathrm{J}=7.9) ; \delta 7.85: 2 \mathrm{H}, \mathrm{d}(\mathrm{J}=7.8) ; \delta 7.58: 2 \mathrm{H}, \mathrm{m} ; \\
\delta 7.47: 1 \mathrm{H}, \mathrm{t}(\mathrm{J}=7.6) ; \delta 7.26: 1 \mathrm{H}, \mathrm{s} ; \delta 7.16: 1 \mathrm{H}, \mathrm{t}((=7.9) .\end{array}$ \\
\hline $\begin{array}{l}\mathrm{GD}_{2} \Rightarrow \delta 7.77: 1 \mathrm{H}, \mathrm{t}(\mathrm{J}=7.3) ; \delta 7.51: 2 \mathrm{H}, \mathrm{d}(\mathrm{J}=7.2) ; \delta 7.27: 3 \mathrm{H}, \mathrm{t}(\mathrm{J}=7.1) ; \delta 7.06: 1 \mathrm{H}, \mathrm{t}(\mathrm{J}=6.7) ; \delta 6.75: 1 \mathrm{H}, \mathrm{d} \\
(\mathrm{J}=7.99) ; \delta 6.49: 1 \mathrm{H}, \mathrm{m} ; \quad \delta 4.08: 1 \mathrm{H}, \mathrm{s} \text { (broad); } \delta 1.62: 2 \mathrm{H}, \mathrm{s} \text { (broad). }\end{array}$ \\
\hline $\mathrm{GD}_{3} \Rightarrow \delta 8.22: 7 \mathrm{H}, \mathrm{dd}(\mathrm{l}=7.1 ; \approx 2) ; \delta 7.55: 7 \mathrm{H}, \mathrm{dd}(\mathrm{l}=7.1 ; \approx 2) ; \quad \delta 7.29: 1 \mathrm{H}, \mathrm{s}$ \\
\hline $\begin{array}{l}\mathrm{GD}_{4} \Rightarrow \delta 8.29: 1 \mathrm{H}, \mathrm{s} ; \delta 7.95: 6 \mathrm{H}, \mathrm{d}(\mathrm{J}=8.7) ; 87.67: 6 \mathrm{H}, \mathrm{d}(\mathrm{l}=8.7) ; \delta 7.40: 1 \mathrm{H}, \mathrm{d}(\mathrm{J}=8.7) ; \delta 6.93: 1 \mathrm{H}, \mathrm{d} \\
(\mathrm{J}=8.7) ; \delta 3.39: 2 \mathrm{H}, \mathrm{s} \text { (broad) }\end{array}$ \\
\hline $\begin{array}{l}\mathrm{GD}_{5} \Rightarrow \delta 7.9: 1 \mathrm{H}, \mathrm{d}(\mathrm{J}=2) ; \delta 7.8: 1 \mathrm{H}, \mathrm{d}(\mathrm{J}=2) ; \delta 7.73: 1 \mathrm{H}, \mathrm{dd}(\mathrm{J}=6.9 ; 1.3) ; \delta 7.51: 5 \mathrm{H}, \mathrm{m} ; \delta 7.33: 2 \mathrm{H}, \mathrm{d} \\
(\mathrm{J}=10.85) ; \delta 7.15: 2 \mathrm{H}, \mathrm{d}(\mathrm{J}=9.725) ; \delta 6.75: 3 \mathrm{H}, \mathrm{m} ; \delta 4.53: 4 \mathrm{H}, \mathrm{m} ; \delta 3.76: 4 \mathrm{H}, \mathrm{m} .\end{array}$ \\
\hline $\mathrm{GD}_{2 \mathrm{~A}} \Rightarrow \delta 8.20: 2 \mathrm{H}, \mathrm{d}(\mathrm{J}=8.6) ; \delta 7.59: 2 \mathrm{H}, \mathrm{d}(\mathrm{J}=8.6) ; \delta 4.55: 2 \mathrm{H}, \mathrm{s}$ \\
\hline $\begin{array}{l}\mathrm{GD}_{7} \Rightarrow \delta 7.76: 3 \mathrm{H}, \mathrm{d}(\mathrm{J}=7) ; \delta 7.53: 4 \mathrm{H}, \mathrm{m} ; \delta 7.27: 1 \mathrm{H}, \mathrm{s} ; \delta 7.05: 1 \mathrm{H}, \mathrm{t}(\mathrm{J}=7) ; \delta 6.74: 1 \mathrm{H}, \mathrm{d}(\mathrm{J}=7) ; \delta 6.49: 3 \mathrm{H}, \\
\mathrm{m} ; \delta 3.71: 2 \mathrm{H}, \mathrm{s} \text { (broad). }\end{array}$ \\
\hline $\begin{array}{l}\mathrm{GD}_{8} \Rightarrow \delta 8.28: 1 \mathrm{H}, \mathrm{d}(\mathrm{V} \approx 8) ; \delta 7.73: 4 \mathrm{H}, \mathrm{m} ; \delta 7.34: 5 \mathrm{H}, \mathrm{m} ; \delta 6.82: 1 \mathrm{H}, \mathrm{m} ; \delta 6.37: 3 \mathrm{H}, \mathrm{t}(\mathrm{l} \approx 8) ; \delta 6.20: 1 \mathrm{H}, \mathrm{d} \\
(\mathrm{j} \approx 6) ; \delta 3.58: 2 \mathrm{H}, \mathrm{s} \text { (broad). }\end{array}$ \\
\hline $\begin{array}{l}\mathrm{GD}_{9} \Rightarrow \delta 7.915: 1 \mathrm{H}, \mathrm{d}(\mathrm{J}=6) ; \delta 7.698: 2 \mathrm{H}, \mathrm{m} ; \delta 7.48: 5 \mathrm{H}, \mathrm{m} ; \delta 7.20915: 2 \mathrm{H}, \mathrm{s} ; \delta 7.1978: 1 \mathrm{H}, \mathrm{t} \\
(\mathrm{J}=11.72) ; \delta 6.8125: 1 \mathrm{H}, \mathrm{dd}(\mathrm{J}=7.7 ; 1.3) ; \delta 6.65: 1 \mathrm{H}, \mathrm{m} ; \delta 6.43: 1 \mathrm{H}, \mathrm{m} ; \delta 6.18: 1 \mathrm{H}, \mathrm{m} ; \delta 4.1378: 1 \mathrm{H}, \\
\mathrm{m} ; \delta 3.6466: 2 \mathrm{H}, \mathrm{m} ; \delta 3.363: 2 \mathrm{H}, \mathrm{m} ; \delta 3.06: 1 \mathrm{H}, \mathrm{m} ; \delta 2.8602: 1 \mathrm{H}, \mathrm{m} ; \delta 1.1941: 1 \mathrm{H}, \mathrm{s}\end{array}$ \\
\hline $\begin{array}{l}\mathrm{GD}_{11} \Rightarrow \delta 8.09625: 2 \mathrm{H}, \mathrm{d}(\mathrm{l}=8.1) ; \delta 7.815475: 2 \mathrm{H}, \mathrm{d}(\mathrm{l}=8.05) ; \\
\delta 7.6278: 3 \mathrm{H}, \mathrm{m} ; \delta 7.39855: 3 \mathrm{H}, \mathrm{m} ; \delta 7.21415: 4 \mathrm{H}, \mathrm{m} ; \delta 6.8865: 1 \mathrm{H}, \mathrm{m} ; \delta 4.31: 1 \mathrm{H}, \mathrm{d}(\mathrm{J}=15.31) ; \delta 3.9366: 1 \mathrm{H} \text {, } \\
\mathrm{d}(\mathrm{J}=15.36) .\end{array}$ \\
\hline $\begin{array}{l}\mathrm{GD}_{12} \Rightarrow \delta 8.535: 3 \mathrm{H}, \mathrm{s} ; \delta 8.3: 2 \mathrm{H}, \mathrm{s} ; \delta 7.64: 4 \mathrm{H}, \mathrm{m} ; \delta 7.125: 3 \mathrm{H}, \mathrm{m} ; \delta 6.65: 2 \mathrm{H}, \mathrm{m} ; \delta 3.8: 3 \mathrm{H}, \mathrm{m} ; \delta 2.5675: 1 \mathrm{H}, \mathrm{d} \\
(\mathrm{J}=18.75) ; \delta 1.13: 1 \mathrm{H}, \mathrm{d}(\mathrm{J}=17.29) .\end{array}$ \\
\hline $\begin{array}{l}\mathrm{GD}_{13} \Rightarrow \delta 7.84: 5 \mathrm{H}, \mathrm{m} ; \delta 7.27: 8 \mathrm{H}, \mathrm{m} ; \delta 6.74: 2 \mathrm{H}, \mathrm{m} ; \delta 4.01: 1 \mathrm{H}, \mathrm{m} ; \delta 3.75: 1 \mathrm{H}, \mathrm{m} ; \delta 3.60: 1 \mathrm{H}, \mathrm{d} \\
(=12) ; \delta 3.20: 1 \mathrm{H}, \mathrm{d}(\mathrm{l}=12) ; \delta 2.45: 1 \mathrm{H}, \mathrm{m} ; \delta 2.25: 1 \mathrm{H}, \mathrm{m} ; \delta 1.75: 2 \mathrm{H}, \mathrm{m} ; \delta 1.50: 1 \mathrm{H}, \mathrm{m} ; \delta 1.25: 2 \mathrm{H}, \mathrm{m} .\end{array}$ \\
\hline
\end{tabular}

\section{Results and Discussion}

The morpholine based sulfonamides were successfully synthesized and their structure well elucidated. These compounds are being tested for the desired activity in our pharmacology division in the department.

\section{Acknowledgements}

We kind owe our sincere thanks to Dr. Gang Liu, Dr. Zhonghua Pei, Dr. Jeffrey R. Huth for spending their invaluable time to clear our doubts and sending reprints of their published articles.

We are grateful to The Director and S. Avtar Singh of R.S.I.C., Panjab University, Chandigarh and The Director and Dr. Dinesh Sultan (Scientist) of R.R.L., Jammu who were kind enough in extending the facility for NMR, IR and Mass Spectral Analysis.

\section{References}

1. Cornejo C. J, Winn R K and Harlan J M Antiadhesion Therapy. Adv. Pharmacol. 19973999

2. Hendrickson; Bergeron Tetrahedron letters. 1970345

3. Vogel's Textbook of Practical Organic Chemistry, Fifth Edition.

4. Norman R O C: Principles of Organic Synthesis, $2^{\text {nd }}$ Edition, Chapman \& Hall, London. 677 


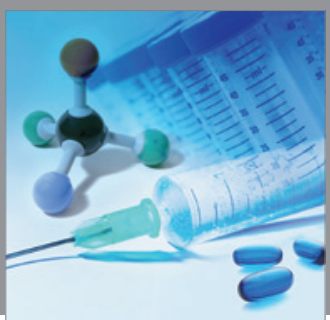

International Journal of

Medicinal Chemistry

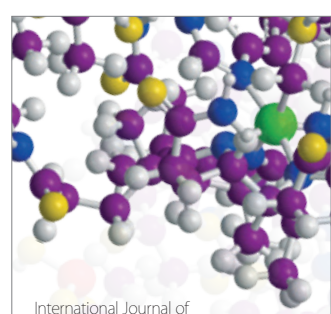

Carbohydrate Chemistry

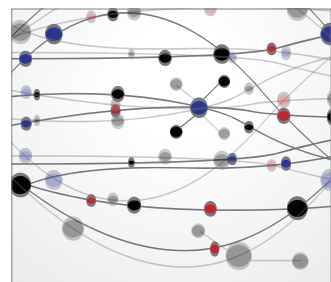

The Scientific World Journal
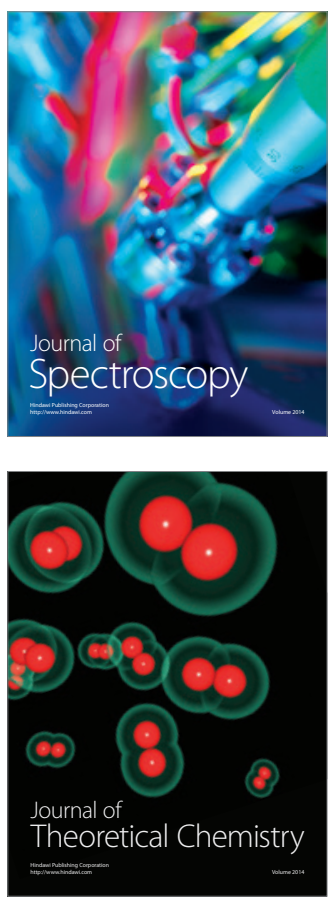
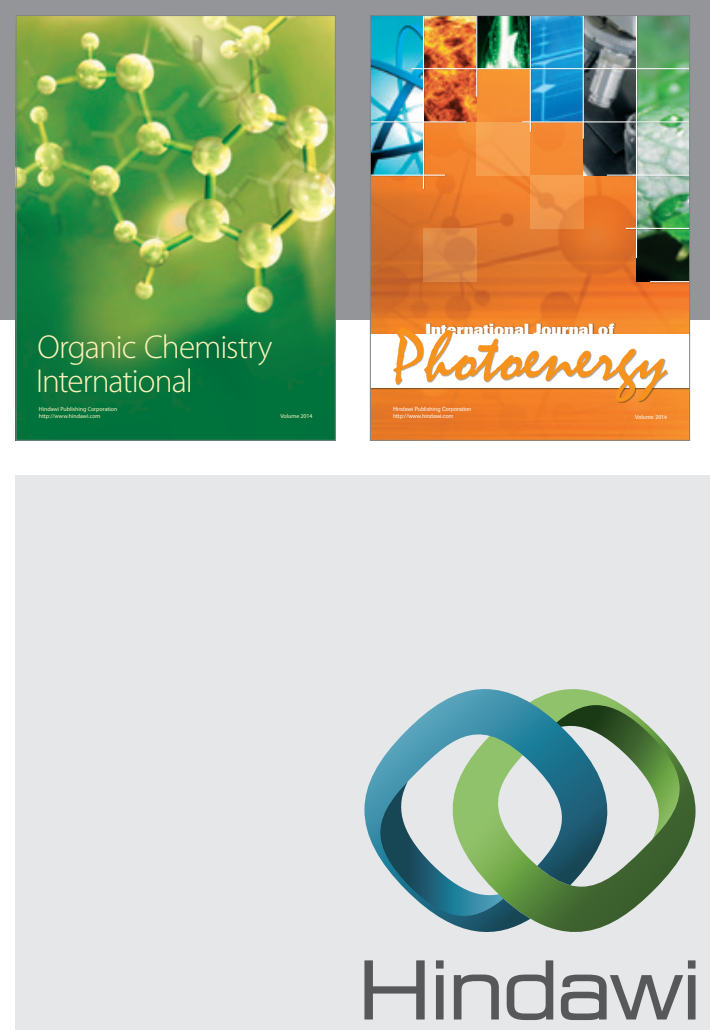

Submit your manuscripts at

http://www.hindawi.com
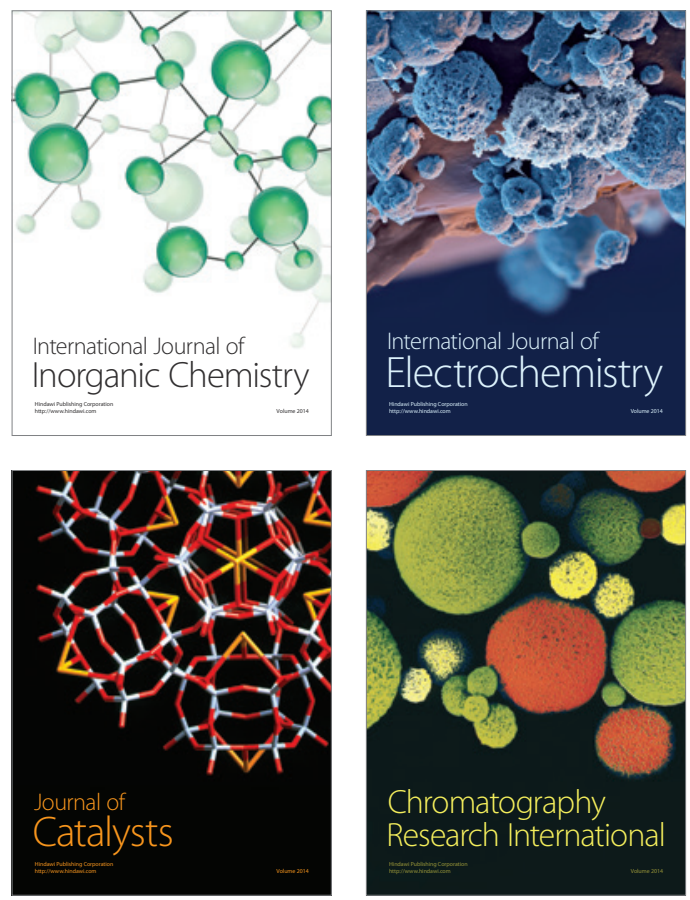
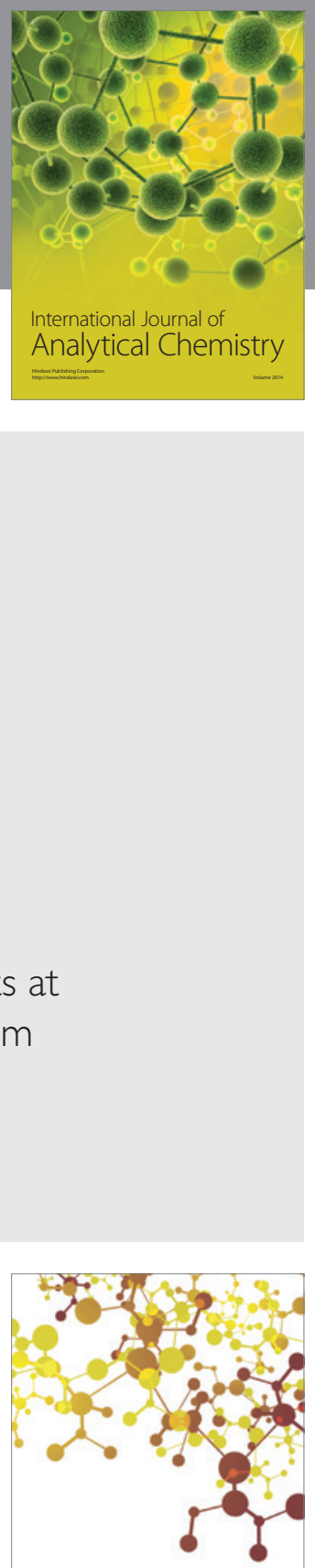

Journal of

Applied Chemistry
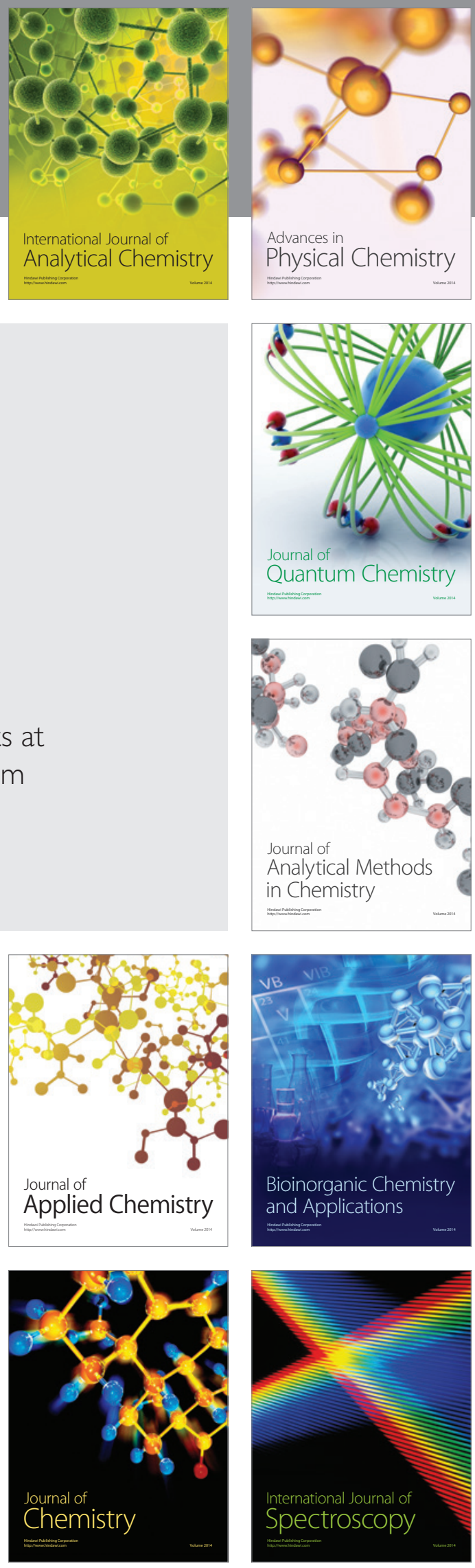